\title{
Factors affecting acceptance of provider-initiated HIV testing and counseling services among outpatient clients in selected health facilities in Harar Town, Eastern Ethiopia
}

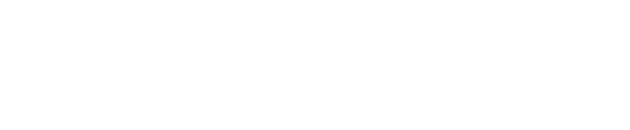

\author{
Sami Abdurahman' \\ Berhanu Seyoum ${ }^{2}$ \\ Lemessa Oljira ${ }^{2}$ \\ Fitsum Weldegebreal ${ }^{2}$ \\ 'Harari Regional Health Bureau, \\ ${ }^{2}$ Haramaya University, College \\ of Health and Medical Sciences, \\ Harar, Ethiopia
}

Correspondence: Fitsum Weldegebreal Haramaya University, College of Health and Medical Sciences, Department of Medical Laboratory Sciences, PO Box 235, Harar, Ethiopia Email fwmlab2000@gmail.com
Purpose: To improve the slow uptake of HIV counseling and testing, the World Health Organization (WHO) and the Joint United Nations Programme on HIV/AIDS (UNAIDS) have developed draft guidelines on provider-initiated testing and counseling (PITC). Both in low- and high-income countries, mainly from outpatient clinics and tuberculosis settings, indicates that the direct offer of HIV testing by health providers can result in significant improvements in test uptake. In Ethiopia, there were limited numbers of studies conducted regarding PITC in outpatient clinics. Therefore, in this study, we have assessed the factors affecting the acceptance of PITC among outpatient clients in selected health facilities in Harar, Harari Region State, Ethiopia.

Materials and methods: Institutional-based, cross-sectional quantitative and qualitative studies were conducted from February 12-30, 2011 in selected health facilities in Harar town, Harari Region State, Ethiopia. The study participants were recruited from the selected health facilities of Harar using a systematic random sampling technique. The collected data were double entered into a data entry file using Epi Info version 3.5.1. The data were transferred to SPSS software version 16 and analyzed according to the different variables.

Results: A total of 362 (70.6\%) clients accepted PITC, and only $39.4 \%$ of clients had heard of PITC in the outpatient department service. Age, occupation, marital status, anyone who wanted to check their HIV status, and the importance of PITC were the variables that showed significant associations with the acceptance of PITC upon bivariate and multivariate analyses. The main reasons given for not accepting the tests were self-trust, not being at risk for HIV, not being ready, needing to consult their partners, a fear of the results, a shortage of staff, a busy work environment, a lack of private rooms, and a lack of refresher training, which were identified as the main barriers for PITC.

Conclusion: There is evidence of the relatively increased acceptability of PITC services by outpatient department clients. A program needs to be strengthened to enhance the use of PITC; the Ministry of Health, Regional Health Bureau, and other responsible bodies - including health facilities - should design and strengthen information education and communication/behavioral change and communication interventions and promote activities related to PITC and HIV counseling and testing in both health facilities and the community at large.

Keywords: PITC, outpatient clients, Harar town, Eastern Ethiopia

\section{Background}

Globally, at the end of 2008, an estimated 6,800 people were found to be infected with HIV each day, and over 5,700 people died; over 2 million people died that year. ${ }^{1}$ Until recently, the primary model for providing HIV testing and counseling has been 
voluntary counseling and testing (VCT) $;^{2}$ however, uptake of VCT was very low. Based on these concerns, the World Health Organization (WHO) and the Joint United Nations Programme on HIV/AIDS (UNAIDS) have developed draft guidelines on provider-initiated testing and counseling (PITC) in health facilities to improve HIV-related diagnosis and treatment. PITC occurs in health facilities as either a part of routine medical care or for purposes of clinical diagnosis. At least $80 \%$ of the 30 million people living with HIV/AIDS in sub-Saharan countries do not know that they are HIV-positive. ${ }^{3}$ Globally, the new WHO/UNAIDS guidelines advise that health care providers should recommend HIV testing and counseling to all patients who present with conditions that might suggest underlying HIV disease. ${ }^{4}$ The PITC model is often used in health care settings, such as in tuberculosis (TB) clinics and in sexually transmitted infection clinics. PITC may also occur in other inpatient wards, outpatient departments (OPD), and primary care clinics. ${ }^{5}$ Evidence from both low- and highincome countries, mainly from outpatient clinics and TB settings, indicates that the direct offering of HIV testing by health providers can result in significant improvements in test uptake. ${ }^{6}$

Factors that influence the acceptance or refusal of PITC could be characterized as sociodemographic, personal, behavioral, societal, health-seeking behavior, as well as professional and institutional PITC service delivery. However, there was a scarcity of information on PITC in Ethiopia on these aspects. In Ethiopia, there are a limited number of studies that have been conducted regarding PITC in outpatient clinics. Many, but not all, clients accept PITC. The service and client factors need to be known and gain acceptance to improve the service. ${ }^{7}$ Thus, an understanding of these factors could help promote PITC among outpatient clients. Therefore, the aim of this study was to assess the factors that affected the acceptance of health PITC among clients attending outpatient services in selected health facilities in Harar, Ethiopia.

\section{Materials and methods Study area}

This study was conducted in Harari National Regional State governmental health facilities. Harari is one of the regional states of the Federal Democratic Republic of Ethiopia located in the eastern part of Ethiopia at distance of $515 \mathrm{~km}$ from Addis Ababa. It is neighbored to the north by Kombolcha and Jarso, to the east by Gursum and Babile Woreda, to the south by Fedis, and to the west by Haramaya Woreda of the Oromia regional state. The total area of the region is estimated to be $340 \mathrm{~km}^{2}$, of which the proportion of urban areas is $6 \%$ and that of rural areas is $94 \%{ }^{8}$ According to health indicators of Harari region in 1999 performed by WHO, the total population of the region was 209,000 , of which $54 \%$ were urban residents, while the rest resided in the rural parts of the region. ${ }^{9}$ The health service coverage is estimated to be above $100 \%$. There are six hospitals (two military, one private, one nongovernmental, and two governmental) and eight health centers in the region. ${ }^{8}$

\section{Study design and period}

An institution-based, cross-sectional quantitative study complemented with a qualitative study was conducted in three governmental health facilities and one university teaching hospital in Harar, Ethiopia, from February 12-30, 2011.

\section{Sample size estimation and data collection}

The sample size was determined using a single proportion formula, and with the following assumptions: the proportion (P) of OPD clients who accepted the test was $73 \%$; ${ }^{10}$ the confidence level was set at 95\%; a 4\% margin of error was used; and a $10 \%$ nonresponse rate was considered. Thus, the final sample size was 520 clients from the OPD. The study participants were recruited from the selected four health facilities using a systematic random sampling technique. During the study period, if the selected individual was not accepted to participate, the next individual/client who arrived was included. For the quantitative part of the study, structured questionnaires were prepared in English based on information from the available literature, ${ }^{11-14}$ and the questionnaires were translated into Amharic and Oromiffa, and then back to English, to check for their consistency; they were pretested for clarity and cultural acceptability within the town. Participants were interviewed in their local languages by trained data collectors who speak the local languages. Each interview was performed face to face.

For the qualitative part of the study, four focus group discussions (FGDs) were conducted. The rationality of conducting FGD is to get an idea regarding the factors surrounding not utilizing PITC, and to find out if there are additional opinions or questions that remain unanswered. Each group was composed of eight people. A total of four FGDs were conducted. The focus group participants included those who accepted and refused testing, based on sex proportion of the clients in focus group discussion. All discussions were recorded for later transcription and notes were taken. The supervisor moderated the FGDs, while a nurse who was one of the supervisors was assigned as the note-taker and handled 
the recorder during the FGDs. FGDs were conducted first to get insight and to modify the questionnaire, which was used for the quantitative study.

\section{Data entry and analysis}

After the completion of data collection, cleaning, editing, and coding were performed; then, the data were entered using Epi Info software version. 3.5.1 and analyzed using SPSS version 16.0. Descriptive statistics were used to compute the means and standard deviations of the continuous variables and the frequency of categorical variables. Bivariate and multivariate logistic regressions were used to assess the effects of the independent variables on the outcome variable, while simultaneously controlling for other potential confounding factors. For the qualitative part, recorded data were transcribed and analyzed manually using open code software.

The correct and incorrect answers were coded as 1 and 0 , respectively. To generate the overall knowledge score, all correct responses were added. Respondents correctly responding to at least five of the six modes of transmission and primary prevention methods of HIV/AIDS questions were considered to have "good knowledge", and correctly responding to at least three of the six modes of transmission and primary prevention methods of HIV/AIDS questions were considered to have "fair knowledge", while those correctly responding to only one or none of the six modes of transmission and primary prevention methods of HIV/AIDS questions were considered to have "poor knowledge".

\section{Ethical consideration}

The study protocol was approved by the Institutional Health Research and Ethical Committee of the College of Health and Medical Sciences, Haramaya University (Harar, Ethiopia). Participants were informed about the objective of the study and they were assured that the confidentiality of the data would be maintained. Informed consent was obtained from all participants prior to data collection.

\section{Results}

\section{Quantitative results}

Sociodemographic characteristics

of the study participants

In this study, a total of 520 participants were included, with a response rate of $98.6 \%$, and female respondents constituted $47.4 \%$. The majority of participants were in the age range of 25-34 years and they belonged to the Amhara ethnic group; the participants were also government employees and followers of Muslim (Table 1).
Table I Sociodemographic characteristics among OPD clients in four selected health facilities in Harar, Ethiopia, 20 I I

\begin{tabular}{|c|c|c|}
\hline Variables & Frequency $(n=5 \mid 3)$ & Percent \\
\hline \multicolumn{3}{|l|}{$\overline{S e x}$} \\
\hline Male & 270 & 52.6 \\
\hline Female & 243 & 47.4 \\
\hline \multicolumn{3}{|l|}{ Age, years } \\
\hline $15-24$ & 163 & 31.8 \\
\hline $25-34$ & 228 & 44.4 \\
\hline $35-44$ & 106 & 20.7 \\
\hline$>45$ & 16 & 3.1 \\
\hline \multicolumn{3}{|l|}{ Occupation } \\
\hline Student & 113 & 22.0 \\
\hline Government employee & 136 & 26.5 \\
\hline Unemployed & 38 & 7.4 \\
\hline Merchant & 82 & 16.0 \\
\hline Farmer & 41 & 8.0 \\
\hline Housewife & 61 & 11.9 \\
\hline Other* & 42 & 8.2 \\
\hline \multicolumn{3}{|l|}{ Educational level } \\
\hline No formal education & 101 & 19.7 \\
\hline Only read and write & 71 & 13.8 \\
\hline Elementary & 65 & 12.7 \\
\hline Secondary & 136 & 26.5 \\
\hline University or higher & 140 & 27.3 \\
\hline \multicolumn{3}{|l|}{ Ethnicity } \\
\hline Amhara & 190 & 37.0 \\
\hline Oromo & 161 & 31.4 \\
\hline Muslim & 256 & 49.9 \\
\hline Orthodox & 211 & 41.1 \\
\hline Protestant & 34 & 6.6 \\
\hline Catholic & 12 & 2.4 \\
\hline \multicolumn{3}{|l|}{ Marital status } \\
\hline Single & 224 & 43.6 \\
\hline Married & 238 & 46.4 \\
\hline Widowed & 23 & 4.5 \\
\hline Separated & 38 & 5.5 \\
\hline
\end{tabular}

Note: *Other occupation: daily laborer; weaver.

Abbreviations: OPD, outpatient department; n, number.

\section{Knowledge, risk perceptions, awareness, and source of information among OPD clients toward HIVIAIDS and PITC}

The majority ( $87.1 \%$ ) of the respondents mentioned that HIV can be transmitted by unprotected sex. The remaining participants identified that sharing contaminated sharp materials with a person who is already infected with the virus, as well as mother-to-child transition, were other modes of transmission. On the other hand, $14.8 \%$ had at least one misconception about the mode of transmission of HIV, including eating and drinking with infected individuals. Concerning the clients' knowledge of the methods of prevention, the majority $(86.0 \%)$ of the study subjects mentioned abstinence, followed by being faithful $(77.6 \%)$ and using condoms $(77.6 \%)$ as prevention methods. Of the total respondents, $358(68.6 \%), 119(23.2 \%)$, 
and 67 (8.2\%) had good knowledge, fair knowledge, and poor knowledge about HIV/AIDS modes of transmission and prevention methods, respectively (Table 2).

Among the total respondents, only 202 (39.4\%) heard that PITC is part of the OPD service, and their main common sources of information were health facilities (51.5\%) followed by neighbors (19.3\%), friends (17.3\%) and media (11.9\%). The level of perceived was very low among the study participants. Of the total respondents, 369 (71.9\%) did not feel at risk of contracting HIV (Table 2).

Most (70.6\%) of the study participants have been tested for PITC. The reasons why they had not decided to get tested included self-trust, not being at risk, not being ready, and a fear of the results (Figure 1).

The majority (81.2\%) of the study participants believed that the PITC service is necessary. Approximately $27.1 \%$ of the participants responded that the time to check for PITC has been used at any time. However, approximately $25 \%$ indicated that the PITC service should be used only when one wants to know about his/her own results, and $8 \%$ mentioned that it should be used when one is sick (Figure 2).

\section{Key findings of the FGD Knowledge about HIVIAIDS transmission and prevention}

Most participants knew of the ways of HIV/AIDS transmission, prevention methods, and the importance of HIV testing. They explained that HIV can be transmitted by sexual intercourse, unsafe sex, sharp materials, and via mother-

Table 2 Knowledge, risk perceptions, awareness, and source of information among OPD clients toward HIVIAIDS and PITC in Harar, Ethiopia, 201 I

\begin{tabular}{|c|c|c|}
\hline Variables & Frequency & Percent \\
\hline \multicolumn{3}{|c|}{ Knowledge of HIV/AIDS $(n=5 \mid 3)$} \\
\hline Good knowledge & 358 & 68.6 \\
\hline Fair knowledge & 119 & 23.2 \\
\hline Poor knowledge & 41 & 8.2 \\
\hline \multicolumn{3}{|c|}{ Perceived risk of HIV/AIDS $(n=5 \mid 3)$} \\
\hline Yes & 444 & 86.2 \\
\hline No & 71 & 13.8 \\
\hline \multicolumn{3}{|c|}{ Heard of PITC $(n=5 \mid 3)$} \\
\hline Yes & 202 & 39.4 \\
\hline No & 311 & 70.6 \\
\hline \multicolumn{3}{|c|}{ Main source of information of PITC $(n=202)$} \\
\hline Health facilities & 104 & 51.5 \\
\hline Media & 24 & 11.9 \\
\hline Friends & 35 & 17.3 \\
\hline Neighbors & 39 & 19.3 \\
\hline
\end{tabular}

Abbreviations: OPD, outpatient department; PITC, patient-initiated testing and counseling; $n$, number.

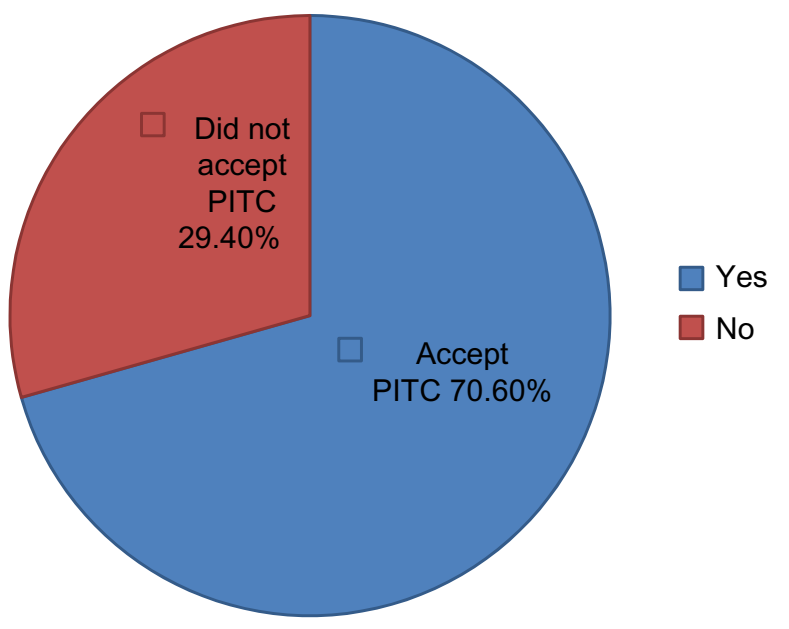

Figure I Percentage distribution of participants tested or not tested for HIV in Harar, Ethiopia, 2011.

Abbreviation: PITC, patient-initiated testing and counseling.

to-child transmission. Most of the participants preferred abstinence and indicated that using condoms was impossible for a long period of time. They also recommended one-toone for those who would attend for marriage people among prevention of HIV/AIDS.

\section{HIV testing and counseling}

Approximately $60 \%$ of the participants did not know about PITC, but all of them knew about VCT. All FGD clients agreed with the importance of PITC services and stated that testing promotes behavioral changes that help the individual avoid risky sexual behavior.

FGD participants felt that the attitude of the community toward people living with HIV was mixed. Half of the participants said that the attitudes toward people living with HIV are good.

Most participants had positive attitudes toward the PITC service. They explained that PITC is important to know one's own status and to plan for marriage. If we are tested, we would change our behavior, and this encourages us to use the service. Most participants agreed to use PITC with their partner.

Most of the FGD participants said that counseling would make them feel more comfortable, would help them accept the test results, release stress and anxiety, and help people change their behaviors and live positively. Most participants proposed that PITC should be obligatory as a premarital test. Females showed more willingness to accept PITC than did males.

\section{Service delivery of PITC}

More than three-quarters of the participants explained that they had not heard about PITC in the OPD clinic or where the PITC services were provided. More than three-quarters of 


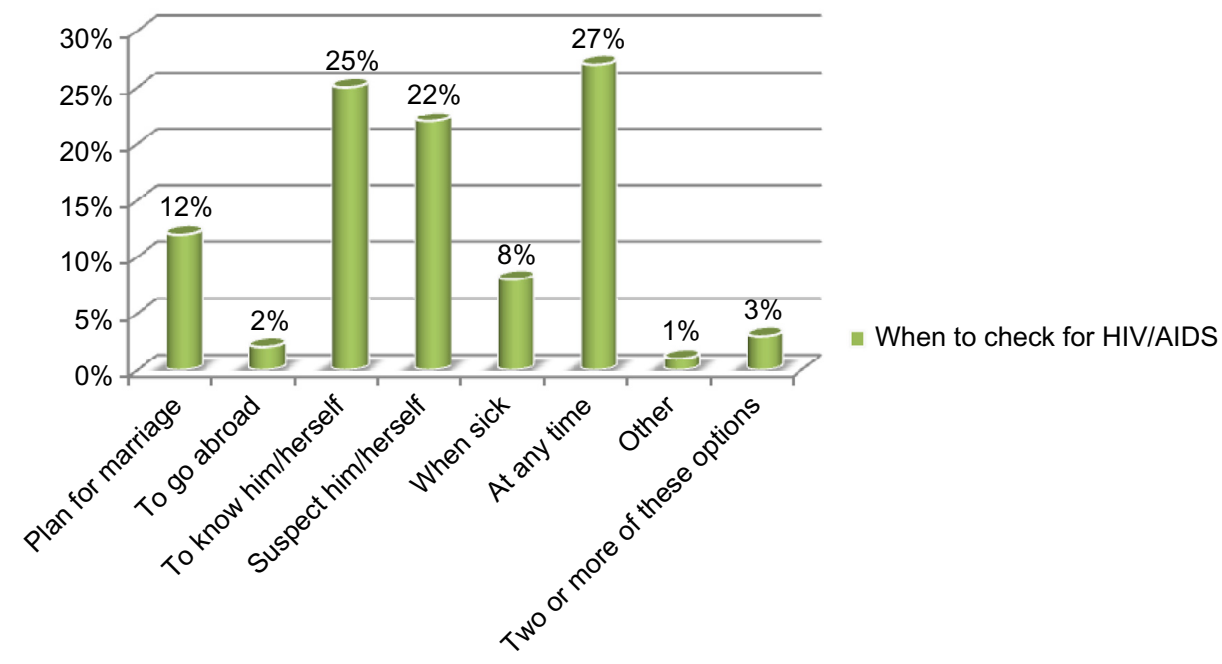

Figure 2 Percentage distribution of when participants think that one should be tested for HIV in Harar, Ethiopia, $201 \mathrm{I}$.

participants also explained that they preferred that PITC services were delivered in hospitals, health centers, and private clinics. They also requested to have free-standing PITC centers, like the VCT center - which is different from the OPD clinic - to maximize client privacy and confidentiality.

A majority of the tested clients in the FGDs said that the reasons why they had decided to be tested included self-interest, initiated by health provider, and knowing that antiretroviral therapy is available. One participant said that, "I want to know my HIV status because I have been engaged in unprotected sex and feel at risk". Two-thirds of FGD participants who decided not to get tested said that they planned to get tested during the next visit to clinic or sometime in the future. The reasons given for not testing on the day included fear of being tested positive, not ready, fear of HIV results, fear of breach of confidentiality, not being at risk, self-trust, inconvenient PITC room, stigma and discrimination, needing to consult partner, do not want to know my status now, and another participant said that "my behavior is good so there is no need for me to be tested".

\section{Results from bivariate and multivariate analyses}

Clients in the age group of 35-44 years were less likely to accept PITC services than those clients whose ages were 15-24 years (adjusted odds ratio [AOR]: 0.17 [0.07-0.41]). On the other hand, clients who had occupations as government employees were more likely to use the PITC services than were student clients (AOR: 5.56 [2.15-14.40]). Clients whose marital status was married were more likely to accept PITC services than were those clients who were single (AOR: 2.85 [1.48-5.46]) (Table 3).
For clients who had felt the importance of PITC in OPD, the service had a positive significant effect on the acceptance of PITC (AOR: 1.35 [1.30-2.63]). Clients who had heard that PITC should be a part of OPD services showed a significant association with the acceptance of PITC (AOR: $0.58 ; P<0.004 ; 95 \%$ confidence interval $[\mathrm{CI}]=0.32-0.91$ ). When clients indicated the importance of PITC in the OPD service, there was a positive and significant effect with the acceptance of PITC (AOR: 1.35; $P<0.001 ; 95 \%$ $\mathrm{CI}=1.30-2.63$ ). Those who were unable to ask clear questions during the discussion yielded a negative and significant effect with the acceptance of PITC (AOR: 0.38; $P<0.001$; $95 \% \mathrm{CI}=0.21-0.68$ ). Finally, clients who did not receive an explanation about confidentiality had a negative significant effect with the acceptance of PITC (AOR $=0.32 ; P<0.001$; $95 \%$ CI $=0.18-0.54)($ Table 4$)$.

\section{Discussion}

The increased uptake of PITC services by OPD clients seems to be due to the fact that PITC has been shown to play a role in health facilities to improve HIV-related diagnosis, treatment, and care. ${ }^{4}$ The PITC service is an integral part of HIV prevention, treatment, care, and support. Ethiopia adopted PITC as one of its HIV/AIDS prevention strategies. The results of this study indicated an acceptability rate of $\sim 70.6 \%$ for PITC by participants. Similar findings were also reported from Dessie, Ethiopia; ${ }^{10}$ Arbaminch, Ethiopia; $;{ }^{11}$ and Addis Ababa, Ethiopia. ${ }^{12}$ However, Weiser et al described a lower acceptability rate of PITC from Massachusetts, United States, which may be due to differences in study times, the start times of PITC, the availability of PITC services, as well as the information education and communication 
Table 3 Sociodemographic factors affecting the acceptance of PITC among OPD clients in Harar, Ethiopia, 20 I I

\begin{tabular}{|c|c|c|c|c|c|}
\hline \multirow[t]{2}{*}{ Variables } & \multicolumn{2}{|c|}{ Acceptance of PITC } & \multirow{2}{*}{$\begin{array}{l}\text { Crude } \\
\text { AOR }(95 \% \mathrm{Cl})\end{array}$} & \multirow{2}{*}{$\begin{array}{l}\text { Adjusted } \\
\text { AOR }(95 \% \mathrm{Cl})\end{array}$} & \multirow[t]{2}{*}{$P$-value } \\
\hline & Yes & No & & & \\
\hline \multicolumn{6}{|l|}{ Sex } \\
\hline Female & $169(69.5)$ & $74(30.5)$ & 1.00 & & \\
\hline Male & $193(7 \mid .5)$ & $77(28.5)$ & $1.10(0.75-1.61)$ & & 0.632 \\
\hline \multicolumn{6}{|l|}{ Age, years } \\
\hline $15-24$ & $122(74.8)$ & $4 I(25.2)$ & 1.00 & & \\
\hline $25-34$ & $166(72.8)$ & $62(27.2)$ & $0.90(0.57-1.42)$ & $0.55(0.26-1.15)$ & \\
\hline $35-44$ & $62(58.5)$ & $44(4 \mid .5)$ & $0.47(0.28-0.80)^{*}$ & $0.17(0.07-0.41)^{*}$ & 0.001 \\
\hline$>45$ & $12(75)$ & $4(25)$ & $\mathrm{I} .0 \mathrm{I}(0.3 \mathrm{I}-3.30)$ & $0.80(0.16-3.96)$ & \\
\hline \multicolumn{6}{|l|}{ Occupation } \\
\hline Student & $69(61.1)$ & $44(38.9)$ & 1.00 & & \\
\hline Government employee & $110(80.9)$ & $26(19.1)$ & $2.70(1.53-4.77)^{*}$ & $5.56(2.15-14.40)^{*}$ & \\
\hline Unemployed & 27 (7I.I) & II (28.9) & $\mathrm{I} .57(0.7 \mathrm{I}-3.47)$ & $6.54(1.98-21.59)$ & \\
\hline Merchant & $56(68.3)$ & $26(31.7)$ & $1.37(0.75-2.50)$ & $3.00(1.11-8.10)$ & \\
\hline Farmer & $28(68.3)$ & $13(31.7)$ & $1.37(0.64-2.93)$ & $5.72(1.69-19.40)$ & \\
\hline \multicolumn{6}{|l|}{ Marital status } \\
\hline Single & $148(66.1)$ & $76(33.9)$ & 1.00 & & $<0.049$ \\
\hline Married & $182(76.5)$ & $56(23.50)$ & $1.67(1.11-2.5 I)$ & $2.85(1.48-5.46)^{*}$ & $0.001(P<0.16)$ \\
\hline Divorced or widowed & $15(65.2)$ & $8(34.8)$ & $0.96(0.39-2.37)$ & $1.88(0.60-5.94)$ & \\
\hline
\end{tabular}

Note: *Statistically significant $(P<0.05)$.

Abbreviations: PITC, provider-initiated testing and counseling; OPD, outpatient department; AOR, adjusted odds ratio; Cl, confidence interval.

program on HIV counseling and testing (HCT).${ }^{15}$ The high acceptability of PITC in our study is promising for scale up of the approach in other settings, which consequently will have an impact on the prevention and control of HIV transmission. ${ }^{15}$
In this study, the rate of acceptance of HIV testing showed a different distribution for different sociodemographic characteristics. The 35-44-year age group was negatively associated with the acceptance of PITC with an AOR of 0.47 $(P<0.001)$. This age category was perceived as not being

Table 4 Knowledge, risk perceptions, awareness, and acceptability among OPD clients toward HIVIAIDS and PITC, as related to factors affecting the acceptance of PITC among clients in Harar, Ethiopia, 20 I I

\begin{tabular}{|c|c|c|c|c|c|}
\hline \multirow[t]{2}{*}{ Variables } & \multicolumn{2}{|c|}{ Acceptance of VCT } & \multirow[t]{2}{*}{ AOR $(95 \% \mathrm{Cl})$} & \multirow{2}{*}{$\begin{array}{l}\text { Adjusted } \\
\text { AOR }(95 \% \mathrm{Cl})\end{array}$} & \multirow[t]{2}{*}{$P$-value } \\
\hline & $\overline{\text { Yes }}$ & No & & & \\
\hline \multicolumn{6}{|c|}{ HIV/AIDS knowledge } \\
\hline Good & $255(72.2)$ & $98(27.8)$ & $1.84(0.95-3.61)$ & & $0.07 \mid$ \\
\hline Fair & $83(69.7)$ & $36(30.3)$ & $1.63(0.78-3.40)$ & & 0.190 \\
\hline Poor & $24(58.5)$ & $17(4 \mid .5)$ & 1.00 & & \\
\hline \multicolumn{6}{|c|}{ Perceived risk of HIVIAIDS } \\
\hline Yes & $109(75.7)$ & $35(24.3)$ & $1.42(0.91-2.20)$ & & 0.122 \\
\hline No & $253(68.6)$ & $115(3 \mid .2)$ & 1.00 & & \\
\hline \multicolumn{6}{|c|}{ Heard of PITC service } \\
\hline Yes & I 57 (77.7) & $45(22.3)$ & $1.80(1.20-2.71)$ & $0.58(0.32-0.91)^{*}$ & 0.004 \\
\hline No & $205(65.9)$ & $106(34.1)$ & 1.00 & & \\
\hline \multicolumn{6}{|c|}{ Feel that PITC service is necessary } \\
\hline Yes & $313(75.2)$ & $103(24.8)$ & $2.97(1.90-4.70)^{*}$ & $1.35(1.30-2.63)^{*}$ & 0.001 \\
\hline No & $49(50.5)$ & $48(49.5)$ & 1.00 & & \\
\hline \multicolumn{6}{|c|}{ Able to ask questions during pretest counseling discussion } \\
\hline Yes & $269(74.6)$ & $83(23.6)$ & 1.00 & & \\
\hline No & $93(57.8)$ & $68(42.2)$ & $0.42(0.28-0.63)^{*}$ & $0.38(0.21-0.68)^{*}$ & 0.001 \\
\hline \multicolumn{6}{|c|}{ Received explanation about confidentiality } \\
\hline Yes & $280(82.1)$ & $61(17.9)$ & 1.00 & & \\
\hline No & $82(47.7)$ & $90(52.3)$ & $0.20(0.13-0.30)^{*}$ & $0.32(0.18-0.54)^{*}$ & 0.001 \\
\hline \multicolumn{6}{|c|}{ Satisfaction with privacy and counseling } \\
\hline Very satisfied & $94(26.0)$ & $5(3.3)$ & 1.00 & & \\
\hline Satisfied & $210(58.0)$ & $70(46.4)$ & $0.16(0.10-0.41)^{*}$ & $0.24(0.10-0.76)^{*}$ & 0.001 \\
\hline
\end{tabular}

Note: *Statistically significant $(P<0.05)$.

Abbreviations: OPD, outpatient department; PITC, provider-initiated testing and counseling; VCT, voluntary counseling and testing; AOR, adjusted odds ratio; Cl, confidence interval. 
at risk when compared with the young age group; this was supported by a previous study conducted in Addis Ababa, Ethiopia, ${ }^{16}$ where age was significantly associated with the acceptance of PITC.

The current study showed the significantly positive acceptance of PITC among government employees, with an AOR of $2.70(P<0.001)$. This group shows the highest test acceptance rate due to improved risk perception, as well as knowledge of HIV and HCT. Government employees presumably had a chance to know this information through different mechanisms, such as their workplace, mainstream HIV interventions, and access to media on the awareness of HIV, and HIV testing and counseling. This result is in line with a study conducted in Arbaminch, Ethiopia, which showed a significant association between government employees and the acceptance of HIV. ${ }^{10}$

Married clients had significantly higher test acceptance rates with an AOR of $1.67(P<0.001)$. This might show the improved risk perception and the better decision-making power of married clients. Clients that are married may have a lower perception of the risks of acquiring HIV infection, assuming that their partners would be mutually faithful. Other studies reported that the acceptance of HIV tests was higher among unmarried clients. ${ }^{17,18}$

The present study found that the majority of participants had a good knowledge of HIV transmission and protection. They viewed the risk of contracting HIV/AIDS as being associated with sexual transmission, mother-to-child transmission, and transmission via sharp materials infected with HIV/AIDS. Although this was statistically not significant, in our study, more than $68.6 \%$ of the respondents were aware of comprehensive HIV/AIDS knowledge. Most FGD participants had knowledge about HIV/AIDS. Similar studies done in Lagos, Nigeria, indicated that the majority (89.9\%) of clients had good knowledge of the mode of HIV transmission. ${ }^{19}$ In another study conducted in a teaching hospital in Nigeria, it was indicated that $65 \%$ of clients had good knowledge, $24 \%$ had fair knowledge, and $11 \%$ had poor knowledge of HIV infection. ${ }^{20}$ Another similar research study conducted in Addis Ababa, Ethiopia, reported the association between the knowledge of HIV transmission and acceptance of HIV tests, though the association was not statistically significant. ${ }^{13}$

The results of this study showed that self-perceived risk for HIV was not statistically significant in association with the acceptance of PITC (AOR: 1.42; 95\% CI =0.91-2.20). This finding was not comparable with the studies done in Arbaminch, Ethiopia, and elsewhere, which identified personal risk perception as one of the predictors for accepting $\mathrm{HCT},{ }^{11,21}$ and a lack of risk perception was mentioned as the main reason why individuals did not decide to undergo PITC by $14.6 \%$ of the nonacceptors. Such behavior would be challenging to the scaling up of HIV testing and counseling.

Clients that were aware of PITC as a part of OPD services had statistically significantly higher test acceptance rates with an AOR of $1.80(P<0.004)$. Having an awareness of PITC as a part of an OPD service is an enabling factor toward the acceptance of PITC. In this study, approximately $60.6 \%$ of participants reported that they were not aware of PITC as a part of OPD services. The main sources of PITC information were health providers, friends, neighbors, mass media, and health facilities. The findings of the current study are similar to those of a study conducted in Gamo Gofa, Ethiopia, where the main sources of PITC information comprised mainly health facilities, mass media, friends, and family members. ${ }^{14}$

Approximately $81.2 \%$ of our clients felt that PITC services are necessary, and this is associated with a significantly positive acceptance of PITC with an AOR of 2.97 $(P<0.001)$. Very similar findings were encountered among the majority of FGD clients, who felt that PITC services are necessary. A similar finding (87.7\%) was also obtained by Samson in his Gama Gofa, Ethiopia study. ${ }^{14}$ This study had also found that when asked about the timing of HIV testing, study participants indicated that they would get tested at any time $(27.1 \%)$, when one wants to know him/herself (25.0\%), when he/she suspects him/herself (25\%), when planning for marriage $(11.3 \%)$, and when one is sick $(11.9 \%)$. This is slightly different when compared to studies conducted previously in Tigray, Ethiopia. ${ }^{22}$ This shows that testing promotes behavioral changes that help the individual avoid risky sexual behavior, that relief is associated with knowing one's HIV status, and that one has the ability to live a better life or live positively after testing, including protecting oneself and his/ her partners.

The perceived facilitators for the acceptance of PITC were self-interest, initiated by a health worker, ensured of confidentiality, and knowing that treatment is available; this was in line with the study conducted in Addis Ababa, Ethiopia, and a study conducted in Botswana. ${ }^{12,15}$ Very similar findings were encountered in the FGDs. This perceived facilitator would promote positive attitudes toward the acceptance of a PITC service.

Approximately 39\% of the respondents did not accept PITC; with respect to these findings, the major cited barriers toward PITC acceptability among the study participants included perception of not being at risk, not ready, self-trust, fear of results, stigma and discrimination, not counseled properly, not sure of confidentiality, needing to consult 
their partners, and being tested before. These were the factors that were identified as why they had not decided to get tested, which is in line with findings from studies conducted in Gama Gofa, Ethiopia; Arbaminch, Ethiopia; Botswana; and Massachusetts, USA. ${ }^{11,14,15,23}$ In addition to confirming the quantitative findings, the FGDs called attention to other important factors: a shortage of staff; workload; a busy working environment; the lack of private rooms; the lack of refresher training; no clear policies; and less and interrupted HIV testing kits supply and provision - these were some of the barriers that affected the acceptance and utilization of PITC. This fact highlights the need for intensive and continuous education about HIV/AIDS and HCT.

Counseling has been identified as a key component of a successful program, which had previously been identified as a problem in Ethiopia. Counseling enables people to make informed decisions about being tested for HIV, assess their personal risk for HIV, and develop risk-reduction strategies. $^{24}$

Pretest counseling is vital for clients to ensure that they understand the implications of negative or positive test results for themselves, their partners, and their family. It is also an opportunity to provide prevention information and individual pretest counseling. It helps patients explore personal HIV risk behaviors and related issues, and offers clarification of the information provided in group sessions. ${ }^{25}$ This study indicated that being unable to ask questions during pretest counseling, not having confidentiality explained to them, and being satisfied with privacy and counseling had significantly negative associations with the acceptance of PITC with an AOR of $0.42(P<0.001)$, AOR of $0.20(P<0.001)$, and AOR of $0.16(P<0.001)$, respectively.

The PITC guidelines indicate that clients expect to receive quality care and counseling from well-trained and competent nurses/counselors with good counseling and interpersonal communication skills, and that only after proper counseling will the client make good decisions. ${ }^{4}$ In the current study, entirely eliminating pretest counseling or providing insufficient information minimizes the opportunities for ensuring informed consent and potentially renders receiving a positive test result more difficult to deal with.

The national PITC guidelines also recommend that the client should be given pretest information on HIV/AIDS and PITC, that the provider must inform the client that he/she has the right to say "no" (to opt out), and that this decision by no means affects the services he or she will get from the health facility. ${ }^{24} \mathrm{WHO} / \mathrm{UNAIDS}$ has responded to these concerns by stating that the implementation of PITC must be accompanied by efforts to create an enabling environment, specified as a health service structure, where HIV testing and counseling are confidential and are accompanied by counseling and informed consent. Staff members are to be adequately trained. The person undergoing the test should be offered or referred to appropriate follow-up services. ${ }^{4}$

\section{Conclusion}

Generally, PITC has been shown to play a crucial role in health facilities to improve HIV-related diagnosis, treatment, and care. In this study, a total of $70.6 \%$ clients were accepting the use of a PITC service. Those factors related to a high rate of acceptance of PITC services include self-interest, initiation by a health provider, knowing that antiretroviral therapy is available, and knowing one's HIV status, while those factors responsible for not accepting PITC include not being at risk, self-trust, not ready, stigma and discrimination, not counseled properly, not sure of confidentiality, needing to consult their partners, being tested before, and fearing the results.

Increasing awareness using creative/promotional activities, the provision of information education and communication activities, advertisement in mass media, and integration of the service in all health institutions are some of the improvements that are needed to change the perception of the community and clients to expand the availability and uptake of provider-initiated HIV testing and counseling in clinical settings. Counseling, confidentiality, and a consent policy should be put in place at all PITC sites to safeguard the interests of the clients. More training is needed on PITC and rapid HIV testing for all levels of health personnel providing these services in OPD and other health service departments in all health facilities. In addition, further studies are needed regarding the quality of PITC in clinical settings from the perspectives of service providers.

\section{Acknowledgments}

We are grateful to the study participants. The study was financially supported by Haramaya University, College of Health and Medical Sciences, Harar, Ethiopia.

\section{Author contributions}

SA designed the study; participated in data collection, analysis, interpretation, and write-up; drafted the manuscript; and critically revised the manuscript. BS participated in the study design and critically revised the manuscript. LO participated in the study design and critically revised the manuscript. FW drafted the manuscript and critically revised the manuscript. All authors read and approved of the final manuscript. 


\section{Disclosure}

The authors report no conflicts of interest in this work.

\section{References}

1. World Health Organization. HIV counselling and testing. AIDS epidemic update, Geneva. 2009:8-16.

2. UNAIDS. HIV counselling and testing. 2010:4-23.

3. World Health Organization. HIV counselling and testing. AIDS epidemic update, Geneva. 2008:4-10.

4. World Health Organization; UNAIDS. Guidance on Provider-Initiated HIV Testing and Counselling in Health Facilities. Geneva, Switzerland: World Health Organization; Joint United Nations Programme on HIV/ AIDS; 2007:1-28.

5. Federal Ministry of Health of Ethiopia. Single Point HIV Prevalence Estimate. Adama, Ethiopia: Federal Ministry of Health of Ethiopia; 2007:25-35.

6. Obermeyer CM, Osborn M. The utilization of testing and counseling for HIV: a review of the social and behavioral evidence. Am J Public Health. 2007;97(10):1762-1774.

7. Federal HIV/AIDS Prevention and Control Office. National HIV/AIDS activity report. Addis Ababa, Ethiopia. 2009:5-30.

8. RHB. Regional health physical plan annual report, Harari. 2010.

9. $\mathrm{MOH}$. Health and Health related indicators, Addis Ababa, Ethiopia. 2007. Available from: http://cnhde.ei.columbia.edu/files/5/6/2008-FebFri-102213.pdf. Accessed on June 1, 2011.

10. Netsanet W, Amsalu D. Missed opportunities for earlier HIV testing and diagnosis at the health facilities of Dessie town, Ethiopia. Ethiop Med J. 2008;7(4).

11. Jerene D, Endale A, Lindtjørn B. Acceptability of HIV counseling and testing among tuberculosis patients in south Ethiopia. BMC Int Health Hum Rights. 2007;7:4.

12. Girma S, Enguselassie F. Uptake of provider initiated HIV counseling and testing (PICT) among out patient department (OPD) clients with possible clinical signs of HIV infection in Addis Ababa. Ethiop Med J. 2009;47(4):245-254.

13. Samuel T. Assessment of factor contributing to utilization of provider initiated HIV counseling and testing among women attending ANC in Addis Ababa. 2008; Master Thesis Extract No 12. Available from: www.etpha.org/publications/category/3-extracts.html?...extract-no-12. Accessed on July 1, 2011.
14. Samson T. Assessment of Acceptability of provider initiated HIV counseling and testing among Tuberculosis patients on DOTS in selected areas in Gamo Gofa Zone, SNNPR. 2008; Master Thesis Extract No 13. Available from: www.etpha.org/publications/category/3-extracts. html?...extract-no-13. Accessed July 1, 2011.

15. Weiser SD, Heisler M, Leiter K, et al. Routine HIV testing in Botswana: a population-based study on attitudes, practices, and human rights concerns. PLoS Med. 2006;3(7):e261.

16. Melaku M. Assessment of VCT utilization, and willingness to accept provider initiated HIV counseling and testing among tuberculosis patient in Addis Ababa. 2007: Unpublished.

17. Messelech. Assessment of Intension and Practice of VCT, Infant Feeding in the Context of HIVIAIDS among Lactating Mothers in Harar Town [master's thesis]. Addis Ababa, Ethiopia: Addis Ababa University; 2006. Available from: www.etpha.org/publications/category/3-extracts.html. Accessed Oct 1, 2011.

18. Thior I, Gabaitiri L, Grimes J, et al. Voluntary counseling and testing among post-partum women in Botswana. Patient Educ Couns. 2007;65(3):296-302.

19. Ekanem EE, Gbadegesin A. Voluntary counseling and testing (VCT) for human immunodeficiency virus: a study on acceptability by Nigerian women attending antenatal clinics. Afr J Reprod Health. 2004;8(2):91-100.

20. Gilks CF. The clinical challenge of the HIV epidemic in the developing world. Lancet. 1993;342(8878):1037-1039.

21. Collini P. Opt-out HIV testing strategies. BMJ. 2006. Available from: http://clinicalevidence.bmj.com/downloads/2.Opt-out HIV testing strategies.pdf. Accessed October 5, 2011.

22. Bayray A. Knowledge, attitude, and practice of voluntary counseling and testing for HIV among university students, Tigray, Northern Ethiopia. Momona Ethiopian Journal of Science. 2010;2(1):108-118.

23. Samet JH, Winter MR, Grant L, Hingson R. Factors associated with HIV testing among sexually active adolescents: a Massachusetts survey. Pediatrics. 1997;100(3 Pt 1):371-377.

24. Federal HIV/AIDS Prevention and Control Office, Federal Ministry of Health. Guidelines for HIV Counseling and Testing in Ethiopia. Addis Ababa, Ethiopia: Federal HIV/AIDS Prevention and Control Office, Federal Ministry of Health; 2007.

25. UNAIDS. HIV counselling and testing. 2008:1-17. Available from: www.unaids.org. Accessed Feburary 1, 2011.
HIV/AIDS - Research and Palliative Care

\section{Publish your work in this journal}

HIV/AIDS - Research and Palliative Care is an international, peerreviewed open-access journal focusing on advances in research in HIV, its clinical progression and management options including antiviral treatment, palliative care and public healthcare policies to control viral spread. The journal welcomes original research, basic science,

\section{Dovepress}

clinical \& epidemiological studies, reviews \& evaluations, expert opinion \& commentary, case reports \& extended reports. The manuscript management system is completely online and includes a very quick and fair peer-review system. Visit http://www.dovepress.com/ testimonials.php to read real quotes from published authors. 